\title{
Impact of musculoskeletal co-morbidity of neck and upper extremities on healthcare utilisation and sickness absence for low back pain
}

\author{
W IJzelenberg, A Burdorf
}

Occup Environ Med 2004;61:806-810. doi: 10.1136/oem.2003.011635

\begin{abstract}
See end of article for authors' affiliations ....................

Correspondence to: Dr W IJzelenberg, Department of Public Health, Erasmus MC University Medical Center Rotterdam, PO Box 1738, 3000 DR Rotterdam, Netherlands; w.ijzelenberg@ erasmusmc.nl
\end{abstract}

Accepted 12 May 2004

\begin{abstract}
Aims: To describe the presence of musculoskeletal co-morbidity of the neck and upper extremities among industrial workers with low back pain, and to examine whether it has an impact on healthcare utilisation and sickness absence for low back pain.

Methods: A self administered questionnaire was used to collect data from 505 industrial workers (response 86\%).

Results: The 12 month prevalence of low back pain was 50\%. Among subjects with low back pain the 12 month prevalence of musculoskeletal co-morbidity of the neck and upper extremities was $68 \%$. Among workers with low back pain, subjects with high pain intensity or disabling low back pain were more likely to have musculoskeletal co-morbidity. In comparison to the subjects who report back pain only, subjects with co-morbidity showed worse general health and health related quality of life. No impact of upper extremity co-morbidity was found on healthcare utilisation, and sickness absence due to low back pain. Conclusions: This study provides no evidence that musculoskeletal co-morbidity of the neck and upper extremities influences the choice to seek care or take sick leave due to low back pain among industrial manual workers. For occupational health practitioners the finding of a high co-morbidity is important to consider when implementing workplace interventions aimed at the reduction of specific musculoskeletal complaints, since the controls for one musculoskeletal complaint may impact adversely on another musculoskeletal complaint. Researchers who perform low back pain intervention studies using generic health measures, should take into account the impact of musculoskeletal co-morbidity on these measures.
\end{abstract}

M usculoskeletal complaints are an important cause of morbidity and disability in Western industrialised societies. Low back pain is the most common musculoskeletal complaint in the general population, with, in the Netherlands, a one year prevalence rate of $44 \% .^{1}$ The same national study showed a one year prevalence of $31 \%$ for neck complaints, $30 \%$ for shoulder complaints, $11 \%$ for elbow complaints, and $18 \%$ for complaints of the wrist. Given these high prevalence rates, it is not surprisingly that a subject with low back pain often has experienced other musculoskeletal complaints as well. Several studies have shown that subjects often report more than one musculoskeletal complaint, and musculoskeletal co-morbidity varied between $37 \%$ and $66 \% .^{1-5}$ However, almost all studies on musculoskeletal complaints address only complaints of a specific anatomical region, such as back pain or shoulder pain.

There is circumstantial evidence that among subjects with low back pain musculoskeletal co-morbidity may have a considerable impact on healthcare utilisation and sick leave. Nordin and colleagues ${ }^{6}$ found that workers with low back pain and concurrent musculoskeletal complaints were more likely to remain work disabled than those with low back pain alone. Another study showed that low back pain subjects with a non-disabling co-morbidity were more likely to have sought care for their back pain, whereas those with musculoskeletal (disabling) co-morbidity less often sought care for their back pain. ${ }^{7}$ However, Molano and colleagues ${ }^{4}$ reported that musculoskeletal co-morbidity did not influence care seeking behaviour among construction workers with low back pain in the past 12 months. Although there seems to be a considerable coexistence between back pain and pain experienced in other anatomical regions, the consequences of musculoskeletal co-morbidity on healthcare utilisation and sickness absence for low back pain are not well understood. A greater understanding is essential; for instance, for clinical practice it is important to know whether musculoskeletal co-morbidity influences the decision to seek treatment for low back pain. For researchers performing low back pain intervention studies it is relevant to know whether musculoskeletal co-morbidity may influence their results. Furthermore, for research on the aetiology of back pain it is significant to understand whether back pain complaints are a separate and distinctive entity or just a reflection of a more general musculoskeletal pain syndrome. ${ }^{18}$

The aim of this cross sectional study was to describe the presence of musculoskeletal co-morbidity of the neck and upper extremities among industrial workers with low back pain, and to examine whether it has an impact on healthcare utilisation and sickness absence for low back pain.

\section{METHODS}

\section{Study population and data collection}

In this cross sectional study, the study population consisted of industrial workers who were recruited from nine companies located throughout the Netherlands. The workers participated in a trial on the effects of ergonomic training in combination with in-company physical therapy. A total of 590 employees were invited to participate in the study. All subjects performed physically demanding work and comprised assembly workers, order pickers in warehouses, and maintenance workers at a stevedoring company and a petrochemical plant. If the worker was willing to participate, an informed consent was signed and a self administered questionnaire was filled out during work time. One of the members of the research team was present to help respondents fill out the questionnaire when needed. Selected 


\section{Main messages}

- The 12 month prevalence of musculoskeletal comorbidity of the neck, shoulder, elbow, wrist, and hand was $68 \%$ among industrial workers with low back pain.

- Among workers with low back pain, subjects with high pain intensity or disabling low back pain are more likely to have musculoskeletal co-morbidity.

- No impact of upper extremity co-morbidity was found on healthcare utilisation and sickness absence for low back pain.

\section{Policy implications}

- For occupational health practitioners the finding of a high co-morbidity is important to consider when implementing workplace interventions aimed at the reduction of specific musculoskeletal complaints, since the controls for one musculoskeletal complaint may impact adversely on another musculoskeletal complaint.

- Researchers who perform low back pain intervention studies using generic health measures, should take into account the impact of musculoskeletal co-morbidity on these measures. workers on vacation or sick leave were asked to fill out the questionnaire as soon as possible after return to work. Nonresponders were sent a reminder after two weeks, and a second reminder with questionnaire after three weeks to the home address. A total of 505 workers completed the self administered questionnaire, yielding a response of $86 \%$. Prior to the commencement of the study we received approval for its conduct from the Medical Ethics Committee.

\section{Questionnaire}

The questionnaire contained questions on individual characteristics, low back pain and other musculoskeletal complaints, general health status, sickness absence, and healthcare utilisation. The questions on individual data included age, gender, weight, height, involvement in sports, marital status, and education. ${ }^{9}$ Education of 10 years or less of primary school and lower vocational level was classified as lower educational level, and education at lower general secondary or intermediate vocational level as medium level of education. The body mass index (BMI) was calculated as weight $(\mathrm{kg})$ divided by the square of the height $(\mathrm{m})$; a BMI of 26 or more was considered as overweight.

\section{Low back pain}

We used the standardised Nordic Questionnaire for the nature and severity of musculoskeletal complaints. ${ }^{10}$ Subjects were presented a drawing with a pre-shaded area indicating the area below the lower ribs and above the gluteal folds, and asked whether they had experienced pain or discomfort for at least a day during the past 12 months. Subjects with low back pain were asked to rate their mean pain intensity in the past 12 months from 0 to 10 on a numerical rating scale (NRS). On the NRS, 0 represented no pain at all, and 10 pain as bad as it could be. ${ }^{11}$ The $75 \%$ centile was taken as the cut off point. Chronic complaints were defined as pain which was present almost every day in the preceding 12 months, with a minimal presence for at least three months. ${ }^{12}$

The Roland-Morris Disability Questionnaire was used as a condition specific health status measure for low back pain, designed to measure the presence of 24 activity limitations on a dichotomous scale. A sum score was calculated by adding up the number of negative items, which may range from 0 (no disability) to 24 (maximum disability). ${ }^{13}$ The median score was taken as cut off point.

\section{General health status}

We measured general health with the SF12 ${ }^{14}$ and the EuroQol. ${ }^{15}$ The SF12 is a generic measure of health, which is derived from the SF36. The SF12 has a good test-retest reliability $(r>0.76)$ and a good validity (median $r=0.67){ }^{14}$ It yields two summary scores, the physical component summary scale (PCS12) and the mental component summary scale (MCS12). Both scores may range from 0 to 100, with higher scores representing better health.

In the EuroQol 5, ${ }^{15}$ five dimensions are used as a measurement for preference based health related quality of life. The preference scores for each worker were calculated using weights for different health states (EQ5d) as obtained from a general population in the UK. ${ }^{16}$ Respondents also recorded their health on a visual analogue scale (EuroQol-VAS), somewhere between 0 (worst imaginable health state) and 100 (best imaginable health state). The EuroQol instrument has a good test-retest reliability ${ }^{17}$ and a good validity. ${ }^{18}$ The EQ5D summary measure has shown significant positive correlations with the PCS12 score $(r=0.55)$ and the MCS12 score $(r=0.41)$, and appeared to be slightly less sensitive than the SF12 to differences associated with less severe morbidity. ${ }^{19}$

\section{Sickness absence and healthcare utilisation}

The question on the occurrence of sickness absence was a modified question derived from a study on the reliability of questions on prevalence, frequency, and duration of sickness absence due to back pain. ${ }^{20}$ The questionnaire showed a good agreement for back pain absence (Cohen's $\kappa=0.65$ ).

Healthcare utilisation was measured by a dichotomous variable (yes/no), which described whether a general practitioner, a specialist, or a physical therapist was consulted for low back pain in the past 12 months. ${ }^{4}$ The specialist category includes neurologists, neurosurgeons, and orthopaedic surgeons.

\section{Musculoskeletal co-morbidity}

The occurrence of upper extremity complaints was assessed using the Nordic Questionnaire. ${ }^{10}$ Subjects were asked if they had experienced pain or discomfort in the neck region for at least a day during the past 12 months, again using a preshaded area to define the particular area. Similar questions with pre-shaded areas were asked for complaints in the shoulder region and in the elbow-wrist-hand region.

Musculoskeletal co-morbidity was defined as the presence of complaints in the neck, or shoulder, or elbow-wrist-hand region in the previous 12 months among subjects with low back pain. Since the neck, shoulders, and arms operate as a functional unit, we grouped complaints in these regions together into the category "upper extremity complaints". Chronic neck complaints referred to pain, which was present almost every day in the neck region in the preceding 12 months with a minimal presence for at least three months. Similar definitions were used for chronic pain in the shoulder, and elbow-wrist-hand region. 


\section{Statistical methods}

In the statistical analysis differences between continuous variables were tested with the unpaired Student's $t$ test, since all continuous variables were distributed normally. The differences between frequencies of categorical variables were tested with the $\chi^{2}$ test. Logistic regression analysis was performed to study associations between low back pain characteristics and co-morbidity, as well as healthcare utilisation and co-morbidity. All variables were dichotomised before being entered into the logistic models. The protocol for the analysis consisted of three steps. Firstly, all independent variables were analysed in a univariate model. Secondly, the variables with a $p$ value equal or less than 0.10 were included in a multivariate model by the step forward procedure. The variable with the lowest $\mathrm{p}$ value was put in the model first, followed by the next lowest, and so on. Variables with a p value lower than 0.05 remained in the model and the other variables were excluded. Thirdly, we determined whether all non-significant variables were excluded correctly by including them in the multivariate model of step two. When one of the odds ratios changed more than $10 \%$, the variable was included in the multivariate model of step two. The analyses were carried out with the statistical package SAS 8.2. ${ }^{21}$

\section{RESULTS}

\section{Subjects and musculoskeletal complaints}

The majority of the study population was male $(94.2 \%)$, married $(83.7 \%)$, had a mean age of 41.5 (SD 9.8) years, and a body mass index of 26.2 (SD 4.4). Most of the workers had a low $(42.9 \%)$ or medium level of education $(46.7 \%)$.

Table 1 shows the 12 month prevalence of low back pain and upper extremity co-morbidity. The prevalence for low back pain in the previous 12 months was $49.9 \%(\mathrm{n}=252)$. A total of $277(54.9 \%)$ of the 505 employees reported complaints in one or more anatomical regions of the upper extremities. The larger part of upper extremity complaints was located in the shoulder (31.2\%) and neck (27.1\%).

Musculoskeletal co-morbidity was high. Among workers with back pain in the past 12 months, 170 (67.5\%) subjects reported concurrent complaints in the upper extremities. The coexistence of low back pain and musculoskeletal complaints in the upper extremities was higher than expected on basis of independence. Low back pain was associated with pain in the neck (OR 2.66, 95\% CI 1.76 to 4.01), shoulder (OR 2.21, 95\% CI 1.50 to 3.26 ), elbow-wrist-hand (OR $1.76,95 \%$ CI 1.14 to 2.69 ), and any upper extremity complaints (OR 2.83, 95\% CI 1.97 to 4.07$)$. Among subjects with chronic low back pain $(\mathrm{n}=36)$, other chronic complaints were common. Chronic low back pain was associated with chronic neck pain (OR $4.81,95 \%$ CI 1.48 to 15.57 ), chronic shoulder pain (OR 2.62,

Table 1 The 12 month prevalence of low back pain and upper extremity co-morbidity among blue collar workers

\begin{tabular}{|c|c|c|}
\hline & $\mathbf{n}$ & $\%$ \\
\hline \multicolumn{3}{|l|}{ All workers $(n=505$ ) } \\
\hline Low back pain & 252 & 49.9 \\
\hline Upper extremity complaints & 277 & 54.9 \\
\hline Neck pain & 137 & 27.1 \\
\hline Shoulder pain & 158 & 31.3 \\
\hline Elbow, and/or wrist, hand pain & 112 & 22.2 \\
\hline \multicolumn{3}{|l|}{ Workers with low back complaints ( $\mathrm{n}=252$ ) } \\
\hline Low back pain and upper extremity complaints* & 170 & 67.5 \\
\hline Low back pain and neck pain* & 92 & 36.5 \\
\hline Low back pain and shoulder pain* & 100 & 39.7 \\
\hline Low back pain and elbow and/or wrist, hand pain* & 68 & 27.0 \\
\hline
\end{tabular}

95\% CI 0.89 to 7.73 ), and chronic complaints of elbow-wristhand (OR 7.12, 95\% CI 1.48 to 34.39). Chronic low back pain was significantly associated with the occurrence of chronic upper extremity complaints (OR 3.59 CI 1.47 to 8.80 ).

\section{Determinants of low back pain and concurrent upper extremity complaints}

Table 2 summarises associations of low back pain characteristics and upper extremity musculoskeletal co-morbidity (LBP with UE). Subjects with high pain intensity or disabling low back pain were more likely to have musculoskeletal comorbidity; however, sciatica was not associated with comorbidity. Chronic back pain in the past 12 months showed an increased risk on concurrent upper extremity complaints, but this association did not reach the conventional level of significance. The score on the Roland Morris Disability Questionnaire for the low back pain subjects with upper extremity co-morbidity was $21 \%$ higher (mean 4.80 , SD 4.98 ) compared with subjects with low back pain only (mean 3.97, SD 5.05).

None of the individual characteristics age, BMI (OR 1.51, 95\% CI 0.88 to 2.59 ), marital status (living alone OR 0.90, $95 \%$ CI 0.38 to 2.11 ), gender (male OR $0.42,95 \%$ CI 0.12 to 1.52), educational level (lowest level OR 1.73, 95\% CI 0.69 to 4.36 ), or participation in sports (OR $0.74,95 \%$ CI 0.44 to 1.26) did determine the occurrence of upper extremity co-morbidity.

\section{General health status}

Table 3 summarises the impact of upper extremity comorbidity on general health and health related quality of life. Subjects who reported only low back pain (LBP only) or only upper extremity complaints (UE only) had lower scores on the PCS12 and EQ5d compared with subjects without complaints. Subjects with back pain and musculoskeletal upper extremity co-morbidity (LBP with UE) showed scores very similar to an additive effect of back pain and of upper extremities complaints.

\section{Healthcare utilisation and sickness absence}

A total of 112 workers (44.4\%) with low back pain consulted a healthcare provider in the past 12 months and approximately a third $(32.9 \%)$ went on sick leave at least once for their back complaints. Table 4 shows that upper extremity comorbidity had no impact on healthcare utilisation or on sickness absence due to low back pain. Use of care from a healthcare provider was determined by disabling low back pain (OR 3.27, 95\% CI 1.89 to 5.67) and high pain intensity (OR 2.51, 95\% CI.1.34 to 4.70). None of the individual characteristics was associated with healthcare utilisation. Low back pain subjects with chronic musculoskeletal co-morbidity sought less often care for their back pain and more often for these other musculoskeletal complaints.

Table 2 Odds ratios (OR) of the association between low back pain characteristics and the occurrence of upper extremity musculoskeletal co-morbidity (LBP with UE) in the past 12 months among industrial workers with low back pain $(n=252)$

\begin{tabular}{|c|c|c|c|}
\hline \multirow[b]{2}{*}{ Low back pain characteristics } & \multirow[b]{2}{*}{$\mathbf{n}$} & \multicolumn{2}{|c|}{ LBP with UE $(n=170)$} \\
\hline & & $\overline{O R}$ & $95 \% \mathrm{Cl}$ \\
\hline High pain intensity & 66 & $1.91^{*}$ & 1.00 to 3.66 \\
\hline Sciatica & 51 & 0.81 & 0.42 to 1.55 \\
\hline $\begin{array}{l}\text { Back pain lasting more than } 3 \\
\text { months }\end{array}$ & 36 & $2.22^{* *}$ & 0.93 to 5.32 \\
\hline High perceived disability & 106 & $1.73^{*}$ & 1.00 to 3.00 \\
\hline
\end{tabular}


Table 3 Impact of only low back pain (LBP only), only upper extremity complaints (UED only), and low back pain with musculoskeletal upper extremity co-morbidity (LBP with UE) on general health (PCS12, MCS12) and health related quality of life (EQ5d and EuroQolVas) compared to those without low back pain or upper extremity complaints (no MSD) among industrial workers $(n=505)$

\begin{tabular}{|c|c|c|c|c|c|}
\hline & $\mathbf{n}$ & $\begin{array}{l}\text { PCS12 } \\
\text { Mean (SD) }\end{array}$ & $\begin{array}{l}\text { MCS12 } \\
\text { Mean (SD) }\end{array}$ & $\begin{array}{l}\text { EQ5d } \\
\text { Mean (SD) }\end{array}$ & $\begin{array}{l}\text { EuroQol VAS } \\
\text { Mean (SD) }\end{array}$ \\
\hline \multicolumn{6}{|l|}{ LBP only } \\
\hline No MSD & 146 & $53.36(5.26)$ & $54.30(6.91)$ & $0.96(0.08)$ & $83.40(15.01)$ \\
\hline Yes & 82 & $49.05(8.20)$ & $55.81(6.34)$ & $0.83(0.19)$ & $80.12(16.26)$ \\
\hline$\Delta$ & & $4.31(6.47)^{*}$ & $-1.51(6.71)$ & $0.13(0.13)^{*}$ & $3.27(15.49)$ \\
\hline \multicolumn{6}{|l|}{ UE only } \\
\hline No MSD & 146 & $53.36(5.26)$ & 54.30 (6.91) & $0.96(0.08)$ & $83.40(15.01)$ \\
\hline Yes & 107 & $50.76(6.66)$ & $53.30(6.27)$ & $0.89(0.14)$ & 80.55 (11.92) \\
\hline$\Delta$ & & $2.60(5.90)^{*}$ & $1.02(6.64)$ & $0.07(0.11)^{*}$ & 2.84 (13.74) \\
\hline \multicolumn{6}{|l|}{ LBP with UE } \\
\hline No MSD & 146 & $53.36(5.26)$ & 54.30 (6.91) & $0.96(0.08)$ & $83.40(15.01)$ \\
\hline Yes & 170 & $46.67(8.50)$ & $51.89(7.71)$ & $0.79(0.19)$ & 75.16 (14.83) \\
\hline$\Delta$ & & $6.69(7.30)^{*} \dagger$ & $2.41(7.38)^{*} \dagger$ & $0.18(0.15)^{*}$ & $8.23(14.91)^{*} \dagger$ \\
\hline
\end{tabular}

${ }^{*} p<0.05$, Student's $t$ test.

†Significant difference $(p<0.05)$ between subjects with LBP only and subjects with LBP and upper extremity comorbidity.

PCS12, physical component summary scale of SF12; MCS12, mental component summary scale of SF12; EQ5d, preference based health related quality of life on five dimensions of EuroQol, using weights for different health states of each individual worker as obtained from a general population in the UK; EuroQol-VAS, EuroQol-visual analogue scale.

\section{DISCUSSION}

The findings of this study indicate that a substantial proportion of subjects with low back pain experienced musculoskeletal co-morbidity in the past 12 months. Subjects with high pain intensity, or disabling low back pain were more likely to have musculoskeletal co-morbidity. In comparison with subjects who reported back pain only, subjects with co-morbidity showed worse general health and health related quality of life. No impact of upper extremity co-morbidity was found on healthcare utilisation and sickness absence due to low back pain.

Some methodological issues have to be considered when interpreting the results of this study. First, the findings in this study are not importantly biased by non-response since all subjects were from the same occupational populations and our response rate was high $(86 \%)$. Second, the results presented might be biased by the fact that both low back pain and co-morbidity were assessed over a one year recall period. This does not necessarily imply the simultaneous occurrence of low back pain and co-morbidity, but could also indicate an episode of low back pain followed by a separate episode of upper extremity complaints within one year. However, since we found a strong association between chronic complaints and co-morbidity, we assume that this does not substantially affect our results. Third, our results relied on cross sectional data and on data generated from survey research-that is, on information provided by the

Table 4 Musculoskeletal upper extremity co-morbidity and healthcare utilisation and sickness absence due to low back pain among industrial workers $(n=252)$

\begin{tabular}{|c|c|c|c|c|}
\hline & \multicolumn{2}{|c|}{ LBP only ( $n=82$ ) } & \multicolumn{2}{|c|}{ LBP with UE $(n=170)$} \\
\hline & $\mathbf{n}$ & $\%$ & $\mathbf{n}$ & $\%$ \\
\hline Medical care seeking for LBP & 31 & 37.8 & 81 & 47.7 \\
\hline General practitioner & 23 & 28.1 & 60 & 35.3 \\
\hline Medical specialist & 6 & 7.3 & 6 & 3.5 \\
\hline Physical therapist & 19 & 23.2 & 52 & 30.6 \\
\hline Sickness absence due to LBP & 26 & 31.7 & 57 & 33.5 \\
\hline
\end{tabular}

LBP only, only low back pain.

LBP with UE, low back pain with musculoskeletal upper extremity comorbidity. respondent about healthcare utilisation and sickness absence. Recall of medical events can be telescoped forward in time, which is most likely to occur with major or traumatic events. ${ }^{22}$ In contrast, some types of care, such as physicians' visits, may not be recalled by respondents, yielding an underestimation of utilisation. ${ }^{23}$ Results might be different when data from other databases, for example, medical records or company records, are used. Sickness absence might be underreported because of socially desirable answers. ${ }^{20}{ }^{24}$ Using sick leave data collected in a standardised way from the employers' registration system may have yielded different sickness absence data, but in this branch of industry absence registers are often incomplete, especially for specific musculoskeletal complaints. Finally, since we used cross sectional data the healthy worker effect could be present, hence underestimating healthcare utilisation and sickness absence.

The 12 month prevalences of particular musculoskeletal complaints were within the range of reported prevalences in other occupational populations with physically demanding jobs. ${ }^{3}{ }^{4}$ The coexistence of low back pain and musculoskeletal complaints in the upper extremities was significantly higher than expected on the basis of independence. We found a 12 month prevalence of co-morbidity of $68 \%$ among subjects with low back pain. Previous studies reported prevalences of co-morbidity varying between $37 \%$ and $66 \% .^{1-5}$ Comparison is hampered, because various definitions of co-morbidity were used. Musculoskeletal co-morbidity was associated with more serious low back pain complaints; however, we did not find a relation between co-morbidity and sciatica. Even though the symptom sciatica in itself is not particularly accurate in diagnosing a prolapsed disc, this supports the suggestion that non-specific low back pain is more influenced by perceptions of pain and reporting behaviour than low back pain associated with a well defined pathology. Among subjects with chronic low back pain other chronic upper extremity complaints were common (OR 3.59, 95\% CI 1.47 to 8.80). The tendency of clustering of chronic complaints in certain subjects has been reported before and also reflects a more general musculoskeletal pain syndrome. ${ }^{18}$

However, healthcare utilisation was determined by the nature and severity of low back pain and not influenced by musculoskeletal co-morbidity. This is in agreement with a 
previous study among construction workers, ${ }^{4}$ in which no impact of upper extremity co-morbidity was found on healthcare utilisation due to low back pain.

We found that low back pain subjects with severe musculoskeletal co-morbidity sought care less often for their back pain and more often for these other musculoskeletal complaints. Similar results have been reported before by Hurwitz and Morgenstern ${ }^{7}$ in a population based survey among the US adult population.

No significance differences were found for frequency and duration of sickness absence between subjects reporting low back pain and those also reporting concurrent complaints in the upper extremities. In a previous study Nordin and colleagues $^{6}$ found that workers with low back pain and concurrent musculoskeletal complaints were more likely to remain work disabled than those with low back pain alone. An explanation for the difference between results could be a larger sample size and the fact that their database included the exact start and end dates of sickness absence for low back pain subjects, whereas we applied a categorical classification for sickness absence, which is less precise.

The results of our study have clear implications. The majority of medical research on low back pain is limited to the lower back region, and does not take into account the coexistence of pain in other anatomical regions. For these researchers, it is important to know whether their results are influenced by musculoskeletal co-morbidity. Our results imply that researchers, who perform low back pain intervention studies using generic health measures, should take into account the impact of musculoskeletal co-morbidity on these measures. Our data suggest that there is at most a limited contribution of upper extremity co-morbidity on care seeking for low back pain to a general practitioner or for subsequent referral to other healthcare providers. This study also showed that there is no influence of musculoskeletal co-morbidity on sickness absence for low back pain complaints.

Clinicians and occupational health practitioners, who are involved in the management of low back pain, should be aware that musculoskeletal co-morbidity is very common in low back pain patients. For occupational health practitioners the finding of a high co-morbidity is important to consider in workplace interventions, since an intervention aimed at reducing physical load on the back may impact adversely on the risk factors for another musculoskeletal complaint. Furthermore, our results indicate that subjects with low back pain and co-occurrence of upper extremity complaints have worse general health status and lower health related quality of life, than those with complaints of the back only. Consequently, musculoskeletal co-morbidity may slow or interfere with normal recovery from back pain and may affect response to treatment. Indeed, in clinical care guidelines for the management of low back pain a poor physical and mental health status or well being is categorised as a "yellow flag". ${ }^{25}{ }^{26}$ Hence, a low back pain subject presenting himself with musculoskeletal co-morbidity merits extra attention.

We conclude that this study provides no evidence that musculoskeletal upper extremity co-morbidity influences the decision to seek care or take sick leave due to low back pain among industrial manual workers. For occupational health practitioners the finding of a high co-morbidity is important to consider when implementing workplace interventions aimed at the reduction of specific musculoskeletal complaints, since the controls for one musculoskeletal complaint may impact adversely on another musculoskeletal complaint. Researchers who perform low back pain intervention studies using generic health measures should take into account the impact of musculoskeletal co-morbidity on these measures.

\section{Authors' affiliations}

W IJzelenberg, A Burdorf, Erasmus MC, University Medical Center

Rotterdam, Netherlands

\section{REFERENCES}

1 Picavet HS, Schouten JS. Musculoskeletal pain in the Netherlands: prevalences, consequences and risk groups, the DMC(3)-study. Pain 2003; 102:167-78.

2 Cote P, Cassidy JD, Carroll L. The factors associated with neck pain and its related disability in the Saskatchewan population. Spine 2000;25:1 109-17.

3 Burdorf A, Naaktgeboren B, Post W. Prognostic factors for musculoskeletal sickness absence and return to work among welders and metal workers. Occup Environ Med 1998:55:490-5.

4 Molano SM, Burdorf A, Elders LA. Factors associated with medical careseeking due to low-back pain in scaffolders. Am J Ind Med 2001;40:275-81.

5 Hurwitz EL, Morgenstern H. The effects of comorbidity and other factors on medical versus chiropractic care for back problems. Spine 1997;22:2254-64.

6 Nordin M, Hiebert R, Pietrek M, et al. Association of comorbidity and outcome in episodes of nonspecific low back pain in occupational populations. J Occup Environ Med 2002;44:677-84

7 Hurwitz EL, Morgenstern H. The effect of comorbidity on care seeking for back problems in the United States. Ann Epidemiol 1999;9:262-70.

8 Croft P. The epidemiology of pain: the more you have, the more you get. Ann Rheum Dis 1996;55:859-60.

9 Joung $I M$, Kunst $A E$, van Imhoff $E$, et al. Education, aging, and health: to what extent can the rise in educational level relieve the future health (care) burden associated with population aging in the Netherlands? J Clin Epidemiol 2000;53:955-63.

10 Kuorinka I, Jonsson B, Kilbom A. Standard Nordic questionnaires for the analysis of musculoskeletal symptoms. Appl Ergon 1987;18:233-7.

11 Von Korff M, Jensen MP, Karoly P. Assessing global pain severity by selfreport in clinical and health services research. Spine 2000;25:3140-51.

12 Elders LA, Burdorf A. Interrelations of risk factors and low back pain in scaffolders. Occup Environ Med 2001;58:597-603.

13 Roland M, Morris R. A study of the natural history of low-back pain. Part II: Development of guidelines for trials of treatment in primary care. Spine 1983;8:145-50.

14 Ware J Jr, Kosinski M, Keller SD. A 12-ltem Short-Form Health Survey: construction of scales and preliminary tests of reliability and validity. Med Care 1996;34:220-33.

15 The EuroQol Group. EuroQol-a new facility for the measurement of healthrelated quality of life. Health Policy 1990;16:199-208.

16 Dolan P. Modeling valuations for EuroQol health states. Med Care 1997:35:1095-108.

17 Van Agt HM, Essink-Bot ML, Krabbe PF, et al. Test-retest reliability of health state valuations collected with the EuroQol questionnaire. Soc Sci Med 1994;39:1537-44

18 Brazier J, Jones N, Kind P. Testing the validity of the Euroqol and comparing it with the SF-36 health survey questionnaire. Qual Life Res 1993;2:169-80.

19 Johnson JA, Coons SJ. Comparison of the EQ-5D and SF-12 in an adult US sample. Qual Life Res 1998;7:155-66.

20 Burdorf A, Post W, Bruggeling T. Reliability of a questionnaire on sickness absence with specific attention to absence due to back pain and respiratory complaints. Occup Environ Med 1996;53:58-62.

21 SAS. SAS Software, Version 8.2. Cary, NC: SAS Institute, 1999-2001.

22 Loftus E, Marburger W. Since the eruption of Mt St Helens, has anyone beaten you up? Improving the accuracy of retrospective reports with landmark events. Memory and Cognition 1993;11:114-20.

23 Sawyer JA, Earp JA, Fletcher RH, et al. Accuracy of women's self-report of their last Pap smear. Am J Public Health 1989;79:1036-7.

24 Van Poppel MN, de Vet HC, Koes BW, et al. Measuring sick leave: a comparison of self-reported data on sick leave and data from company records. Occup Med (Lond) 2002;52:485-90.

25 CBO. Kwaliteitsinstituut voor de Gezondheidszorg. Richtlijn aspecifieke lage rugklachten. Alphen aan den Rijn: Van zuiden, 2003.

26 Clinical Standards Advisory Group. Back pain. Report of a CSAG committee on back pain. London: HMSO, 1994. 